\title{
Immunoglobulin G4-mediated sclerosing cholangitis as a risk factor for cholangiocarcinoma: A case report
}

\author{
KARIN E. KOOPMAN ${ }^{1}$, ELISABETH BLOEMENA ${ }^{2}$, GEERT KAZEMIER $^{3}$ and MICHAEL KLEMT-KROPP $^{1}$ \\ ${ }^{1}$ Department of Gastroenterology, Northwest Clinics, 1815 JD Alkmaar; \\ Departments of ${ }^{2}$ Pathology and ${ }^{3}$ Surgery, VU University Medical Center (VUMC), 1081 HV Amsterdam, The Netherlands
}

Received March 31, 2016; Accepted August 8, 2016

DOI: $10.3892 / \mathrm{mco} .2016 .1040$

\begin{abstract}
Immunoglobulin (Ig)G4-mediated disease is a systemic autoimmune disease, which occasionally presents solely as sclerosing cholangitis (SC). IgG4-mediated SC is challenging to diagnose, as it may mimic cholangiocarcinoma radiologically, and carcinoma cells may produce $\mathrm{IgG} 4$. The diagnosis of $\mathrm{IgG} 4$-mediated disease is based on histological consensus criteria and response to corticosteroids. In addition to the radiological and histological overlap between IgG4-mediated SC and cholangiocarcinoma, IgG4-mediated SC may be considered as a risk factor for the development of cholangiocarcinoma. We herein present the case of a patient in whom cholangiocarcinoma developed in two lesions previously characterized as IgG4-mediated SC, including a suggested mechanism underlying the contribution of IgG4-mediated SC to the development of cholangiocarcinoma.
\end{abstract}

\section{Introduction}

Immunoglobulin (Ig)G4-mediated disease is a systemic condition, which may manifest as autoimmune pancreatitis type 1 or extrapancreatic disease, such as biliary lesions, sialadenitis, retroperitoneal fibrosis, enlarged celiac and hilar lymph nodes, chronic thyroiditis and interstitial nephritis (1). Less frequently, IgG4-mediated disease presents solely as sclerosing cholangitis (SC) in the intra- and/or extrahepatic bile ducts, with high plasma levels of $\mathrm{IgG} 4$ and narrowing of the common bile duct (CBD) on imaging (2). It is challenging

Correspondence to: Dr Karin E. Koopman, Department of Gastroenterology, Northwest Clinics, Wilhelminalaan 12, 1815 JD Alkmaar, The Netherlands

E-mail: k.e.m.koopman@nwz.nl

Abbreviations: CBD, common bile duct; ERCP, endoscopic retrograde cholangiopancreaticography; IgG4, immunoglobulin G4; IgG4-SC, immunoglobulin G4-mediated sclerosing cholangitis; IL-10, interleukin 10

Key words: immunoglobulin G4-mediated disease, immunoglobulin G4-mediated sclerosing cholangitis, cholangiocarcinoma to distinguish IgG4-mediated SC (IgG4-SC) from cholangiocarcinoma, as IgG4-SC may mimic cholangiocarcinoma clinically and radiologically (2-4). In addition, carcinomas may be accompanied by significant $\mathrm{IgG} 4$ reactions via production of cytokines (5); thus, the presence of IgG4-positive cells in a bile duct biopsy does not exclude cholangiocarcinoma. We herein present such a diagnostically challenging case, in which the patient appeared to have both IgG4-SC and cholangiocarcinoma.

\section{Case report}

Our patient was a 43-year-old female without relevant medical history or use of any medication. The patient presented with jaundice that was first noticed 4 weeks prior to presentation. Physical examination revealed icteric sclerae and skin and a palpable gallbladder without spider naevi, palmar erythema, enlarged liver or spleen, or pain on palpation. The initial laboratory testing revealed a total bilirubin level of $122 \mu \mathrm{mol} / \mathrm{l}$ (normal range, 2-20 $\mu \mathrm{mol} / \mathrm{l}$ ), direct bilirubin of $89 \mu \mathrm{mol} / 1$ (normal range, 0-5 $\mu \mathrm{mol} / \mathrm{l}$ ), aspartate aminotransferase $56 \mathrm{U} / 1$ (normal range, 0-31 U/1), alanine aminotransferase $64 \mathrm{U} / 1$ (normal range, 0-34 U/1), alkaline phosphate $491 \mathrm{U} / 1$ (normal range, 0-120 U/1) and gamma-glutamyltransferase $132 \mathrm{U} / 1$ (normal range, 0-38 U/1). The cancer antigen 19-9 level was not elevated (19 kU/1). Magnetic resonance cholangiopancreatography revealed dilated intrahepatic biliary ducts in the left and right hepatic lobes and intraluminal wall thickening in the proximal CBD over a length of $25 \mathrm{~mm}$, with a 'shouldering' margin (Fig. 1), a finding suspicious for cholangiocarcinoma. A computed tomography (CT) scan indicated a diffusely thickened pancreatic head, a characteristic that may indicate autoimmune pancreatitis, and hypodense lesions in hepatic segments 6 and 7.

An endoscopic retrograde cholangiopancreatography (ERCP) for placement of a CBD endoprosthesis and brush cytology were performed, after which time cholestasis gradually diminished. However, brush cytology was inconclusive. Additional laboratory testing revealed elevated IgG4 levels of $2.92 \mathrm{~g} / 1$ (normal range, 0.08-1.40 g/l). A treatment for IgG4-associated cholangitis with prednisone $40 \mathrm{mg} /$ day for 1 month was prescribed, with a repeat ERCP after the end of treatment. The repeat ERCP revealed a similar narrowing of the CBD and repeat brush cytology showed malignant cells, 

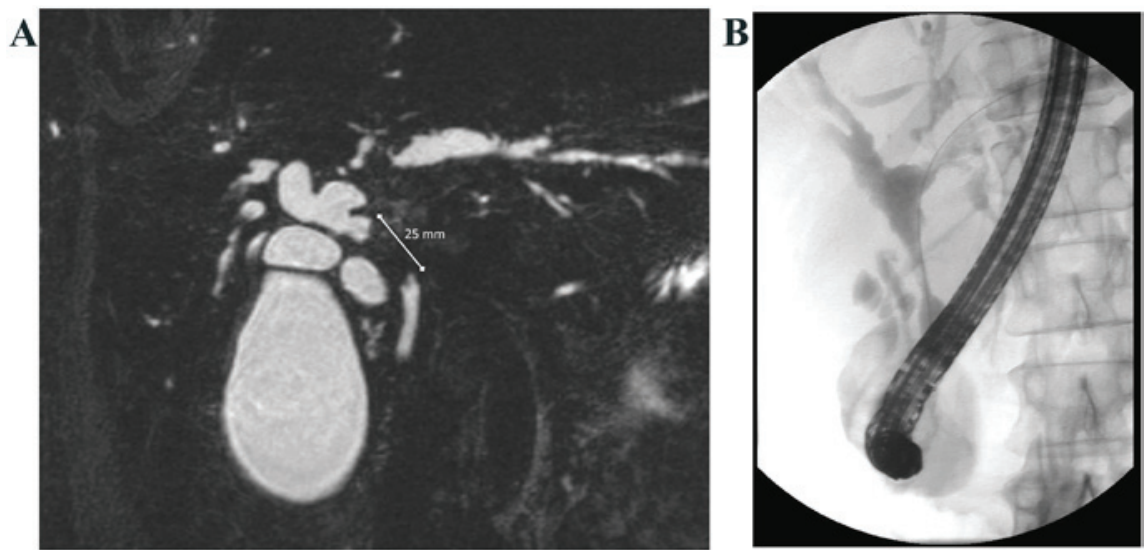

Figure 1. (A) Magnetic resonance cholangiopancreatography at presentation, showing a 25-mm stenosis of the common bile duct with shouldering margins. (B) Endoscopic retrograde cholangiopancreatography after treatment with prednisone, showing stenosis of the common bile duct, comparable with that seen in (A).

A

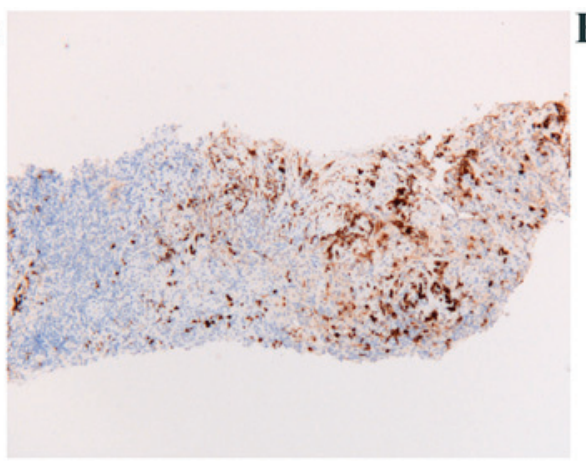

B

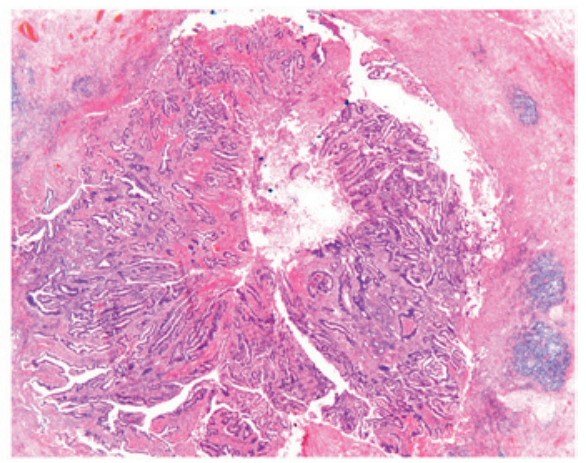

Figure 2. (A) Immunohistochemical staining of the liver biopsy for IgG4, showing a pericholangiolar inflammation with fibrosis, with high concentrations of IgG4-positive cells ( $>20$ per high-power field; magnification, x10). (B) Postoperative histological examination of the common bile duct revealed a T1N0M0 cholangiocarcinoma, as well as IgG4-mediated fibrosis of the common bile duct (hematoxylin and eosin staining; magnification, x5).

suspicious of adenocarcinoma. As there was no response to prednisone and malignant cells were found on repeat brush cytology, IgG4-associated cholangitis became less likely and the patient was referred for surgical treatment for suspicion of a Bismuth type I cholangiocarcinoma. To characterize the lesion in hepatic segments 6 and 7 identified on CT scan(i.e., to exclude metastasis) a liver biopsy was performed. Histological analysis revealed a pericholangiolar inflammation with fibrosis with high concentrations of IgG4-positive cells (>20 per high-power field), and obliterative phlebitis, morphologically matching the diagnosis of IgG4-mediated inflammatory disease. There were no signs of malignancy (Fig. 2A). A laparotomy was next performed, with perioperative histopathological analysis of the CBD stenosis, which revealed a cholangiocarcinoma and was followed by pancreaticoduodenectomy. Postoperative histological examination revealed a T1N0M0 cholangiocarcinoma, as well as IgG4-mediated fibrosis of the CBD (Fig. 2B). The pancreas, duodenum and gallbladder exhibited no abnormalities. The radiological suspicion of autoimmune pancreatitis on an earlier CT scan was therefore not confirmed histologically. The patient was treated with prednisone and azathioprine for the IgG4-mediated disease and her condition improved clinically; however, radiologically, the hypodense lesion in the right hepatic lobe persisted. Repeat biopsy revealed cancer cells and a right hemihepatectomy was performed. Histological analysis of the resected specimen showed a tumor with characteristics identical to the previously diagnosed cholangiocarcinoma. The postoperative course was uncomplicated, but plasma IgG4 levels remained elevated. The patient was treated with prednisone, after which plasma IgG4 levels gradually normalized. The patient remains in remission, as confirmed by 3-monthly follow-up CT scans.

\section{Discussion}

We herein present a case in which histologically proven IgG4-mediated disease was present simultaneously with cholangiocarcinoma. It is known that IgG4-related disease is a high-risk factor for cancer development (6-11). Moreover, primary SC, a disease that clinicopathologically resembles IgG4-SC, is associated with cancer development (12). There is a limited number of case reports of cholangiocarcinoma coexisting with IgG4-SC (13-15) and one case report demonstrated the presence of biliary intraepithelial neoplasia (precursor lesion of bile duct adenocarcinoma) in the postoperative specimen of a patient with IgG4-SC (5); however, a causal association between cholangiocarcinoma and IgG4-SC has not been proven. In our case, the first liver biopsy provided histological proof of IgG4-SC without malignancy, whereas on a later biopsy cancer cells were detected. Similarly, the 
first endoscopic brush cytology of the CBD showed no signs of malignancy, whereas a later brush cytology and the postoperative specimen showed cholangiocarcinoma. Although a sampling error cannot be excluded in both cases, the findings indicate that carcinoma may have developed in an IgG4 lesion. A potential mechanism underlying the contributing role of IgG4-SC to cholangiocarcinoma development is via cytokines: An interleukin-10 (IL-10)-related cytokine milieu initiates the $\mathrm{IgG} 4$ reaction and also suppresses tumor-reactive $\mathrm{T}$ cells, suggesting that IgG4-SC may accelerate the development of cholangiocarcinoma (5). However, the production mechanism of IgG4 in IgG4-related disease has not been fully elucidated; another possibility is that mechanisms contributing to $\operatorname{IgG} 4$ production also contribute to carcinogenesis, rather than IgG4 per se being a causal factor of carcinogenesis (16). However, the simultaneous presence of IgG4-SC and cholangiocarcinoma in two different extrapancreatic lesions in our patient, in addition to previous reports of IgG4-related disease associated with malignancy, is a remarkable finding and suggests at least a pathophysiological association between the two entities.

Extrahepatic cholangiocarcinomas, including gallbladder cancer, are often accompanied by significant IgG4 reactions. The cholangiocarcinoma cells may function as non-professional antigen-presenting cells that indirectly induce IgG4 reactions via the IL-10-producing cells, and/or these may be FOXP3-positive and IL-10-producing cells that directly induce IgG4 reactions (5). However, there are several histomorphological characteristics found in IgG4-related SC that distinguish this condition from malignancy, including a marked degree of bile duct injury, a higher percentage of lymphoid follicle formation, a higher percentage of perineuritis, and a more diffuse and dense lymphoplasmacytic infiltrate (17). These characteristics were present in the liver biopsy of our patient and, therefore, it is unlikely that the IgG4 reaction was induced by carcinoma.

In conclusion, we herein report a case in which two lesions, first characterized as IgG4-SC without signs of malignancy, were later diagnosed as cholangiocarcinoma. This phenomenon has been previously reported in IgG4-SC, and IgG4-related disease in general is closely associated with the development of malignancies. We therefore suggest that IgG4-SC is a risk factor for the development of cholangiocarcinoma. This hypothesis is speculative, since supporting histopathological evidence is not yet available; however, if confirmed, our hypothesis has serious clinical implications. Longitudinal follow-up studies of patients with IgG4-SC, as well as histological examination of IgG4-SC biopsies for carcinoma precursor lesions, cytokine expression and lymphocyte subtyping, may strengthen our hypothesis and it is crucial that such studies are performed in the near future. Moreover, patients with IgG4-SC must be closely monitored and when they no longer respond to immunosuppressive therapy, cholangiocarcinoma should be suspected.

\section{References}

1. Detlefsen S: IgG4-related disease: A systemic condition with characteristic microscopic features. Histol Histopathol 28: 565-584, 2013

2. Lin J, Cummings OW, Greenson JK, House MG, Liu X, Nalbantoglu I, Pai R, Davidson DD and Reuss SA: IgG4-related sclerosing cholangitis in the absence of autoimmune pancreatitis mimicking extrahepatic cholangiocarcinoma. Scand J Gastroenterol 50: 447-453, 2015.

3. Cheung MT and Lo IL: IgG4-related sclerosing lymphoplasmacytic pancreatitis and cholangitis mimicking carcinoma of pancreas and Klatskin tumour. ANZ J Surg 78: 252-256, 2008.

4. Miki A, Sakuma Y, Ohzawa H, Sanada Y, Sasanuma H, Lefor AT, Sata N and Yasuda Y: Immunoglobulin G4-related sclerosing cholangitis mimicking hilar cholangiocarcinoma diagnosed with following bile duct resection: Report of a case. Int Surg 100: 480-485, 2015.

5. Harada K and Nakanuma Y: Cholangiocarcinoma with respect to IgG4 Reaction. Int J Hepatol 2014: 803876, 2014.

6. Fukui T, Mitsuyama T, Takaoka M, Uchida K, Matsushita M and Okazaki K: Pancreatic cancer associated with autoimmune pancreatitis in remission. Intern Med 47: 151-155, 2008.

7. Ghazale A and Chari S: Is autoimmune pancreatitis a risk factor for pancreatic cancer? Pancreas 35: 376, 2007.

8. Inoue H, Miyatani H, Sawada Y and Yoshida Y: A case of pancreas cancer with autoimmune pancreatitis. Pancreas 33: 208-209, 2006.

9. Loos M, Esposito I, Hedderich DM, Ludwig L, Fingerle A, Friess H, Klöppel G and Büchler P: Autoimmune pancreatitis complicated by carcinoma of the pancreatobiliary system: A case report and review of the literature. Pancreas 40: 151-154, 2011.

10. Pezzilli R, Vecchiarelli S, Di Marco MC, Serra C, Santini D, Calculli L, Fabbri D, Rojas Mena B and Imbrogno A: Pancreatic ductal adenocarcinoma associated with autoimmune pancreatitis. Case Rep Gastroenterol 5: 378-385, 2011.

11. Huggett MT, Culver EL, Kumar M, Hurst JM, Rodriguez-Justo M, Chapman MH, Johnson GJ, Pereira SP, Chapman RW, Webster GJ and Barnes E: Type 1 autoimmune pancreatitis and IgG4-related sclerosing cholangitis is associated with extrapancreatic organ failure, malignancy, and mortality in a prospective UK cohort. Am J Gastroenterol 109: 1675-1683, 2014.

12. Mendes F and Lindor KD: Primary sclerosing cholangitis: Overview and update. Nat Rev Gastroenterol Hepatol 7: 611-619, 2010

13. Douhara A, Mitoro A, Otani E, Furukawa M, Kaji K, Uejima M, Sawai M, Yoshida M, Yoshiji H, Yamao J and Fukui H: Cholangiocarcinoma developed in a patient with IgG4-related disease. World J Gastrointest Oncol 5: 181-185, 2013.

14. Oh HC, Kim JG, Kim JW, Lee KS, Kim MK, Chi KC, Kim YS and Kim KH: Early bile duct cancer in a background of sclerosing cholangitis and autoimmune pancreatitis. Intern Med 47: 2025-2028, 2008.

15. Straub BK, Esposito I, Gotthardt D, Radeleff B, Antolovic D, Flechtenmacher C and Schirmacher P: IgG4-associated cholangitis with cholangiocarcinoma. Virchows Arch 458: 761-765, 2011.

16. Kusuda T, Uchida K, Miyoshi H, Koyabu M, Satoi S Takaoka M, Shikata N, Uemura Y and Okazaki K: Involvement of inducible costimulator- and interleukin 10-positive regulatory $\mathrm{T}$ cells in the development of IgG4-related autoimmune pancreatitis. Pancreas 40: 1120-1130, 2011.

17. Deshpande V, Zen Y, Chan JK, Yi EE, Sato Y, Yoshino T, Klöppel G, Heathcote JG, Khosroshahi A, Ferry JA, et al: Consensus statement on the pathology of IgG4-related disease. Mod Pathol 25: 1181-1192, 2012. 